\title{
Transcriptome Analysis to Identify Putative Genes Involved in Flowering Time Under Different Photoperiods in 'Hong jin gou' Common Bean
}

\author{
Xiaoxu Yang ${ }^{1}$ and Chang Liu ${ }^{1}$ \\ Horticulture Department, Academy of Crop Science, Heilongjiang University, 74 Xuefu Road, 150000 \\ Harbin, Heilongjiang, China; Work Station of Science and Technique for Post-doctoral in Sugar Beet \\ Institute Affiliated to Heilongjiang University, 74 Xuefu Road, 150000 Harbin, Heilongjiang, China; \\ and Post-doctoral Research Station Affiliated to Northeast Agricultural University, 59 Mucai Road, \\ 150000 Harbin, Heilongjiang, China
Zhishan Yan, Youjun Fan, Guojun Feng ${ }^{2}$, and Dajun Liu ${ }^{2}$
Horticulture Department, Academy of Crop Science, Heilongjiang University, 74 Xuefu Road, 150000 Harbin, Heilongjiang, China

\begin{abstract}
Additional Index words. Phaseolus vulgaris, day length, RNA-seq, time-to-flower
ABstract. Flowering time influences pod yield and quality of common bean (Phaseolus vulgaris); however, our knowledge of flowering time genes and flowering mechanisms in common bean remain limited. We performed RNAsequencing (RNA-seq) analyses [long-day (LD) condition and short-day (SD) condition] to identify the flowering time genes and analyzed differentially expressed genes to examine their expression levels in relation to flowering time in 'Hong Jin Gou' common bean, a cultivar highly sensitive to photoperiod. The circadian patterns of related genes were identified using quantitative real-time polymerase chain reaction (qRT-PCR). Flowering time in 'Hong Jin Gou' was influenced by day length: SD conditions promoted flowering. A total of eight flowering time-related genes were identified, which were classified into photoperiod pathways. Homologs of pseudo-response regulator 5, pseudoresponse regulator 7 , and gigantea were more highly expressed under SD conditions than under LD conditions. Homologs of late elongated hypocotyl and timing of cab expression 1 were differentially expressed under light and dark conditions. Early flowering 3 is a key regulator of the pathway, which coordinates light and circadian clock inputs in leaves to trigger the expression of downstream genes. The present study provides critical information that could facilitate further investigations on the genetic mechanism of flowering time in common bean.
\end{abstract}

Environmental factors (daylength and temperature) and internal signals (gibberellins and autonomous pathways) simultaneously regulate flowering time in plants (Bhakta et al., 2017; Putterill et al., 2004). Higher plants detect fluctuations in daylength, which influence flowering time with changes in seasons. Common bean is a tropical facultative SD legume that is currently grown in tropical and temperate zones. The observation underscores how domestication and modern breeding practices can alter the adaptive phenology of a species (Moyses et al., 2018). During common bean domestication and dissemination from its centers of domestication, selection for photoperiod insensitivity allowed common bean to spread to higher latitudes (Gepts and Debouck, 1991). In addition, the determinate growth habit has been exploited in crop breeding activities to accelerate flowering and shorten the flowering period (Cober and Tanner, 1995).

Received for publication 17 Apr. 2019. Accepted for publication 9 May 2019. Financial support was provided by the China Postdoctoral Science Foundation (grant number 2018M631973); the basic scientific research operating expenses of provincial College in Heilongjiang province (grant numbers KJCXYB201707 and KJCXYB201705); the National Natural Science Funds of China (grant number 31771869); and the applied technology research and development plan of Heilongjiang province (grant number GY2019YF0059).

${ }^{1}$ These authors contributed equally to this work and share first authorship.

${ }^{2}$ Corresponding authors.

E-mail: jianlongedu@163.com or feng998@126.com.
In comparison with LD plants like Arabidopsis thaliana, much less is known about the genetic mechanisms that regulate flowering in SD plants, such as soybean (Glycine max), rice (Oryza sativa), and maize (Zea mays), among others; however, some progress has been made in such species through genetic analyses that have facilitated the identification of numerous genes. As a first step toward isolating the genes in common bean, we sought to use information obtained in model species such as $A$. thaliana using a candidate gene approach. Photoreceptors, circadian clock components, bio-clock, and lightregulated genes are key components for daylength detection using the external coincidence model. Among the clock and light-regulated genes, constans $(\mathrm{CO})$ has been identified as a key gene participating in the integration of light and clock signals. The overexpression of the $C O$ gene leads to early flowering in $A$. thaliana through the regulation of the expression of downstream genes, such as flowering locust (FT), apetalal (API), and leafy $(L F Y)$, regardless of the length of daylight (Aidyn et al., 2002; Suárez-López et al., 2001; Yanovsky and Kay, 2002).

Intriguingly, more detailed molecular genetics analyses have revealed that several quantitative trait locus (QTL) associated with time-to-flower in SD species are orthologs of genes that regulate flowering in A. thaliana (Lee and An, 2007; Simpson and Dean, 2002; Salome et al., 2011). For example, rice QTL, HD1, and HD3a were found to be orthologs of $A$. thaliana $C O$ and $F T$, respectively (Kojima et al., 2002; Tamaki 
et al., 2007). Such analyses have also discovered some flowering QTL that do not have orthologs in $A$. thaliana, like the early heading date $1 \mathrm{QTL}$ in rice, a gene that regulates the expression of FT (Itoh et al., 2010). The observation suggests that additional mechanisms that control time-to-flower are likely to be discovered in SD plants. Buttressing this point is the discovery that setaria (Setaria viridis), a SD grass, has a secondary mechanism that operates under long days (Doust et al., 2017). RNA-seq technology facilitates the discernment of novel perspectives in transcriptome sequence analysis by providing comprehensive coverage of transcripts. In addition, RNA-seq could be used as an alternative to other transcript quantification approaches with the benefit of higher sensitivity and the potential to distinguish between very similar paralogs of a gene that differ based only on a few nucleotides. RNA-seq has been used to characterize transcriptional changes resulting from different flowering times (Kitae et al., 2017), and the differentially expressed genes (DEGs) identified were found to be useful for predicting differences in tolerance between common bean cultivars. Previous investigations have identified a dominant photoperiod-sensitive gene regulating flowering time in beans ( $\mathrm{Gu}$ et al., 1998; Kornegay et al., 1993; Kwak et al., 2008; Wallace et al., 1991; White and Laing, 1989; White et al., 1996), but a comprehensive genetic analysis of the trait has not been carried out. In the present study, we used RNA-seq and quantitative reverse transcription PCR (qRT-PCR) to investigate potential changes in the common bean transcriptome in response to the changes in daylength. Known genes and novel predicted genes were identified and the functions of the molecules associated with the regulation of flowering time were evaluated. The findings could provide a theoretical basis for the enhancement of the adaptation of common bean cultivars to different photoperiods.

\section{Materials and Methods}

Plant materials and light Treatments. A total of 215 common bean cultivars were sown in Harbin [northeast China (lat. $44^{\circ} 30^{\prime} 24^{\prime \prime} \mathrm{N}$, long. $125^{\circ} 42^{\prime} 41^{\prime \prime} \mathrm{E}$ )] and Sanya [south China (lat. $18^{\circ} 09^{\prime} 34^{\prime \prime} \mathrm{N}$, long. $108^{\circ} 56^{\prime} 30^{\prime \prime} \mathrm{E}$ )] on 21 May and 1 Nov. 2017, respectively (Supplemental Table 1). The flowering times of all cultivars were recorded at both sites and the most photoperiod-sensitive cultivars were screened for use as material in the present study. To eliminate the impact of major environmental factors, the seeds of photoperiod-sensitive common bean cultivars, Hong Jin Gou [indeterminate climbing, yellow pod with red streak (developed at Heilongjiang University, Harbin, China)] were cultivated in climate chambers at $25^{\circ} \mathrm{C}$ under LD conditions (16/8 h light/dark) with 300 $\mu \mathrm{mol} \cdot \mathrm{m}^{-2} \cdot \mathrm{s}^{-1}$ of white light. All of plants were cultivated in this climate chambers at first. Half of the plants were transferred to SD conditions $(8 / 16 \mathrm{~h}$ light/dark) under a similar temperature regimen after the trifoliate leaves emerged. The trifoliate leaves at 0600 and $1400 \mathrm{HR}$ under both conditions at $20 \mathrm{~d}$ after emergence were collected and immediately frozen in liquid nitrogen and stored at $-80^{\circ} \mathrm{C}$. Samples were collected at 20 and $21 \mathrm{~d}$ after emergence at the same sampling time points, with leaf samples from the three independent biological replicates being collected at each sampling time. Samples from the same sampling time points during $2 \mathrm{~d}$ were mixed to minimize errors. A total of 12 RNA libraries were constructed, three for each photoperiod $\times$ sampling time-point combination: LD-L (0600 HR), LD-D (1400 HR), SD-L (0600 HR), and SD-D (1400 HR).

RNA EXTRACTION, SEQUeNCING, AND ASSEMbly. Total RNA was extracted from the leaf tissues using TRIzol reagent (Invitrogen, Carlsbad, CA) according to manufacturer's instructions. Total RNA quantity and purity were analyzed using an automated electrophoresis tool (Bioanalyzer 2100; Agilent Technologies, Santa Clara, CA) and RNA 6000 Nano LabChip Kit (Agilent Technologies) with RNA integrity number >7.0. Following purification, the messenger RNA (mRNA) was fragmented into small pieces using divalent cations under elevated temperature conditions. The cleaved RNA fragments were then reverse-transcribed to create the final complementary DNA (cDNA) library according to the protocol of the mRNASeq sample preparation kit (Illumina, San Diego, CA), the

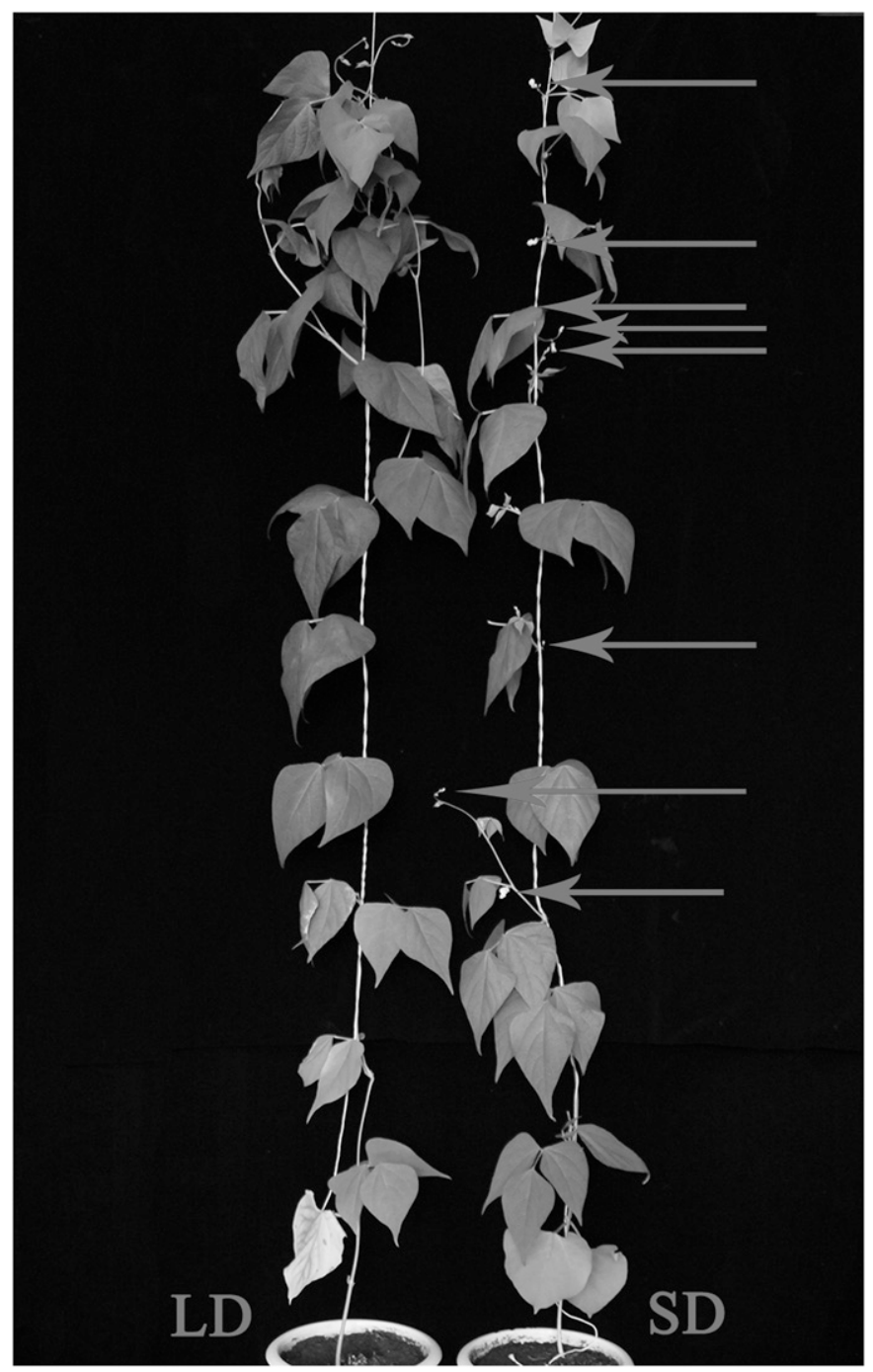

Fig. 1. 'Hong jin gou' common bean [indeterminate climbing, yellow pod with red streak (developed at Heilongjiang University, Harbin, China)] cultivated in climate chambers at $25^{\circ} \mathrm{C}$ under long-day (LD) conditions $(16 / 8 \mathrm{~h}$ light/ dark) with $300 \mu \mathrm{mol} \cdot \mathrm{m}^{-2} \cdot \mathrm{s}^{-1}$ of white light. All plants were cultivated in this climate chamber at first. Half of the plants were transferred to short-day (SD) conditions $(8 / 16 \mathrm{~h}$ light/dark) under a similar temperature regimen after the trifoliate leaves emerged. SD condition promoted flowering in 'Hong jin gou'. The date of flowering initiation was $33 \mathrm{~d}$ after sowing under the SD condition. (The arrows marks the position of flower.) In contrast, under the LD condition, the date of flowering initiation was delayed by $\approx 37 \mathrm{~d}$. 
Table 1. Sequencing and assembly statistics of two time points following long-day [LD (16/8 h light/ dark)] and short-day [SD (8/16 h light/dark)] photoperiod treatment in common bean. Four pools of messenger RNA samples from SD and LD conditions were used to build libraries for highthroughput sequencing. More than 6 billion total nucleotides were obtained from each library, and all high-quality reads were selected and assembled. Each sample from the leaves of the three independent biological replicates for each of sampling times.

\begin{tabular}{lcccc}
\hline Sample identity $^{z}$ & Reads $\left(\times 10^{6}\right)$ & Base pairs $\left(\times 10^{9}\right)$ & Mapped reads $\left(\times 10^{6}\right)$ & Proportion mapped (\%) \\
\hline SD-D & 38.14 & 5.72 & 24.10 & 63.22 \\
SD-L & 43.05 & 6.46 & 28.45 & 66.15 \\
LD-D & 46.59 & 6.97 & 27.53 & 59.12 \\
LD-L & 43.56 & 6.53 & 26.54 & 60.87
\end{tabular}

${ }^{\mathrm{Z}}$ Photoperiod $\times$ sampling time-point combinations: LD-L (0600 HR), LD-D (1400 HR), SD-L (0600 $\mathrm{HR})$, and SD-D (1400 HR).

average insert size for the paired-end libraries was 300 base pairs (bp) $( \pm 50 \mathrm{bp})$. Subsequently, we carried out paired-end sequencing on a HiSEq. 4000, Illumina platform according to the vendor's recommended protocol.

NORMALIZATION OF GENE EXPRESSION LEVELS AND IDENTIFICATION of DEGs. Sequencing reads were aligned to the reference sequences using SOAP aligner/soap 2 (Li et al., 2009). For each gene, the expression level was measured and expressed as reads per kilobase exon model per million map reads (RPKM) based on the number of uniquely mapped reads, to eliminate the influence of different gene lengths and sequencing discrepancies on the gene expression calculation. For genes with more than one alternative transcript, the longest transcript was selected for use in calculating the RPKM.

To infer the transcriptional changes under light conditions, DEGs in the light conditions were identified by comparing the expression levels in LD-L with those in SD-L, and the level in LD-D with those in SD-D (SD as control). To compensate for multiple testing errors, the false discovery rate (FDR) was calculated for use in adjusting the threshold of the probability value (Rajkumar et al., 2015). Transcripts with a minimal 2fold difference in expression $(\mid \log 2$ Ratio $\mid \geq 1)$ and an FDR $\leq$ 0.001 were considered differentially expressed between the two time points. DEGs with higher expression levels in LD-D than in SD-L, as well as those higher in LD-D than in SD-D, were considered "upregulated," whereas those in the exhibiting opposite trends were considered "downregulated."

GENE EXPRESSION VALIDATION. Eight genes with varying expression patterns revealed through RNA-seq were randomly selected for validation by qRT-PCR. RNA extracted from the leaves of the three independent biological replicates of the different sampling times were used for qRT-PCR validation. First-strand cDNA was synthesized using a PrimeScriptRT reagent Kit (Takara Bio, Kusatsu, Japan). Gene copy specific primers for qRT-PCR were designed based on the corresponding sequences using Primer6 (Supplemental Table 2). The qRTPCR was carried out using SYBR Premix Ex TaqII (Takara Bio) and determined in a PCR system (LightCycler 480; Roche, Basel, Switzerland) according to the manufacturer's instructions and the data were collected using method of relative quantification $2-\Delta \Delta \mathrm{Ct}$. Actin was used as the internal control to normalize small differences in template quantities. Three technical replicates were performed for each gene with the aim of obtaining reliable expression results from the RNA-seq. Similar expression patterns were observed in LD-D vs. SD-D and LD-L vs. SD-L when qRT-PCR and RNA-seq data were compared as shown in Supplemental Fig. 1.
FUNCTIONAL ANNOTATION AND GENE ONTOLOGY AND KYOTO ENCYCLOPEDIA OF Genes and Genomes ClassificaTION. BLAST (2.2.3) and BLAST2GO (2.2.5) were used for functional annotation of DEGs, gene ontology (GO) and Kyoto Encyclopedia of Genes and Genomes (KEGG) for pathway enrichment analysis. Significantly enriched GO terms were determined through a hypergeometric test followed by Bonferroni correction to calculate the $P$ value for each term. We established $P<0.05$ as cutoff for a significantly enriched GO term. For the KEGG pathway enrichment analysis, a hypergeometric test followed by an FDR analysis was carried out, and a cutoff of $\mathrm{q}<0.05$ was established for the determination of enriched KEGG pathways.

\section{Results and Discussion}

DIFFERENCE IN TIME-TO-FLOWER UNDER VARIOUS PHOTOPERIOD TREATMENTS. As a typical SD plant, common bean is sensitive to changes in daylength. Photoperiod conditions shorter than $12 \mathrm{~h}$ of light would promote flowering. By observing flowering times in the fields on the plant materials, we observed 'Hong jin gou' was one of the most photoperiod-sensitive cultivars. The flowering initiation times in 'Hong jin gou' were 36 and $69 \mathrm{~d}$ after sowing in Sanya and Harbin, respectively. The results also were verified by laboratory experiments: the SD condition promoted flowering in 'Hong jin gou' (Fig. 1). The time of flowering initiation was $33 \mathrm{~d}$ after sowing in the SD condition. In contrast, in the LD condition, the flowering initiation time was delayed by $\approx 37 \mathrm{~d}$. One of the most visible effects of the plant clock is photoperiodism, the triggering of events such as flowering and senescence in response to the changing seasons (McWatters et al., 2000). In common bean, the major trigger is change in daylength. The results demonstrate that photoperiod had a considerable effect on flowering in 'Hong jin gou'.

RNA-SEQ AND FUNCTIONAL ANNOTATION OF TRANSCRIPTOME. Twelve pools of mRNA samples from the SD and LD condition were used to build libraries for high-throughput sequencing. More than six $G$ nucleotides were obtained from each library and all high-quality reads were selected and assembled. Approximately 38.14 to 46.59 million 125 -bp pair-end reads were generated from the four samples through RNA-seq (Table 1). After sequence trimming, the retained high-quality reads from all the samples were merged into 27,012 genes, and 23,719 of the genes were functionally annotated with an e-value cutoff of $1 \times 10^{-5}$.

Identified DEGs could be pooled into two major groups. Among the 1526 upregulated genes [Fig. 2A(a)], 1155 (61.6\%) were transiently induced only in dark conditions, whereas 545 (29.1\%) were only induced in light conditions, with 174 (9.3\%) displaying increased expression in both conditions. In addition, we observed 1224 DEGs that exhibited downregulation [Fig. $2 \mathrm{~A}(\mathrm{~b})], 57$ (4.3\%) of which exhibited decreased expression in both light and dark conditions, 502 (37.5\%) DEGs that were particularly downregulated under light conditions, and 779 $(58.2 \%)$ that were downregulated in dark conditions [Fig. $2 \mathrm{~A}(\mathrm{~b})]$. 
A

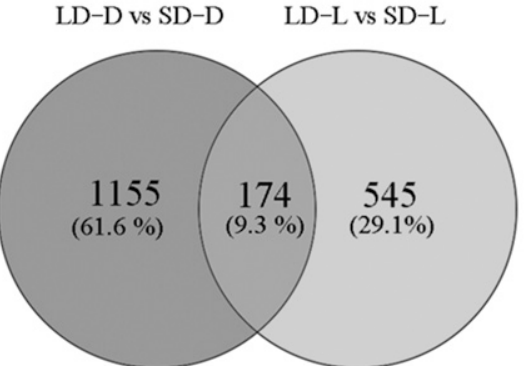

(a)

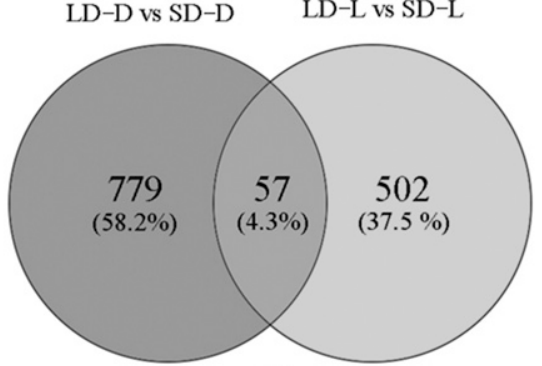

(b)
B

\begin{tabular}{|c|c|c|c|}
\hline Class & GO term & Light & Dark \\
\hline 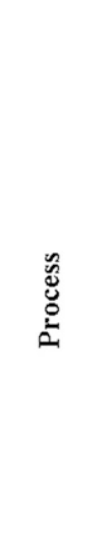 & $\begin{array}{l}\text { circadian rhythm } \\
\text { regulation of circadian rhythm } \\
\text { negative regulation of circadian rhythm } \\
\text { regulation of flower development } \\
\text { rhythmic process } \\
\text { positive regulation of flower development } \\
\text { protein phosphorylation } \\
\text { regulation of auxin biosynthetic process } \\
\text { vegetative phase change } \\
\text { photomorphogenesis } \\
\text { negative regulation of long-day photoperiodism, flowering } \\
\text { auxin homeostasis } \\
\text { positive regulation of long-day photoperiodism, flowering } \\
\text { rhythmic process }\end{array}$ & & \\
\hline 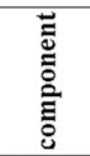 & $\begin{array}{l}\text { chloroplast photosystem II } \\
\text { photosystem II oxygen evolving complex } \\
\text { photosystem I reaction center } \\
\text { chloroplast }\end{array}$ & & \\
\hline 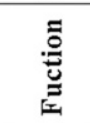 & $\begin{array}{l}\text { chlorophyll binding } \\
\text { transporter activity } \\
\text { protochlorophyllide reductase activity }\end{array}$ & & \\
\hline
\end{tabular}

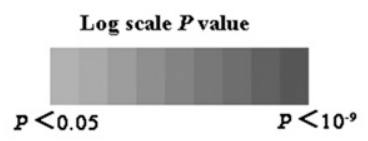

Fig. 2. Gene expression reprogramming under different photoperiod conditions in common bean. (A) Venn diagrams displaying the overlaps among differentially expressed genes identified in common bean plants exposed to light and dark conditions. Numbers in parentheses denote percentages with respect to the total upregulated (a) or downregulated (b) genes; long-day [LD (16/8 h light/dark)] and short-day [SD (8/16 h light/ dark)] photoperiod $\times$ sampling time-point combination [LD-L (0600 HR), LD-D (1400 HR), SD-L (0600 HR), and SD-D (1400 HR)]. (B) Selected gene ontology (GO) terms enriched among differentially expressed genes identified in common bean. Color panels display the probability values of GO term enrichments.

previously, the results of the GO term enrichment analysis highlighted the relevance of transcription factor activity under different photoperiod conditions. Notably, among the 121 DEGs encoding putative transcription factors, whose expression were induced in both dark and light under LD and SD conditions, 15 circadian rhythm genes were observed to be significantly associated with flowering time genes in the current transcriptome data set (Table 2). The findings suggested that flowering time in common bean shares some molecular mechanisms with photoperiod responses in SD plants.

Circadian clocks are biological timing mechanisms used by pluricellular organisms to predicatively adjust physiological and molecular processes in anticipation of environmental changes that occur as a consequence of the day-night cycle, and they are the major factors that regulate flowering time. In the present transcriptomic analysis, to evaluate functions and expression patterns of circadian clock-related genes, we found eight DEGs associated with flowering time, based on an analysis using the KEGG database [early flowering 3 (ELF3), pseudo-response regulator 5 (PRR5), pseudo-response regulator 7 (PRR7), late elongated hypocotyl (LHY), timing of cab expression 1 (TOC1), gigantea (GI), constans (CO), and flowering locus T (FT)] (Table 3). Only LHY and TOCl had different expression patterns under light and dark conditions.

Little is known about flowering time genes and flowering mechanisms in common bean. Although misregulation of several oscillator components results in altered flowering phenotypes, the precise mo-

After functional annotation of the DEGs, we performed GO classification and a GO term enrichment analysis as the first step in understanding the molecular changes that were induced by the different photoperiod conditions. The enrichment analysis revealed that one of the functions that dominated the photoperiod condition (light) was transcription factor activity (Fig. 2B). Other GO terms that were enriched were associated with the circadian rhythm. There was a significant enrichment of DEGs associated with flower development, circadian rhythm, and photosystem.

EXPRESSION PATTERN ANALYSIS OF FLOWERING TIME-RELATED GENES. Photoperiod is a critical factor regulating plant developmental processes, particularly flowering. As mentioned lecular interactions between the circadian clock and photoperiodic flowering remain still poorly understood (Nagel and Kay, 2012). In the present study, we performed highthroughput transcriptome sequencing, revealing 15 flowering time-related genes in photoperiod pathways (Table 2). A total of eight putative flowering time genes were classified into the photoperiod pathways. $L H Y$ and $C C A 1$ repress floral transition under SD and LD conditions by inhibiting the expression of TOC1 in the central loop of the circadian clock, and play partially redundant roles in the control of the circadian clock (Fujiwara et al., 2008; Lu et al., 2009; Zhuo et al., 2010). In this study, compared with the low expression levels of the homolog of $L H Y$, the homolog of TOCl was highly expressed in all 
Table 2. Fifteen genes associated with flowering time in common bean. Among the 121 differentially expressed genes encoding putative transcription factors, whose expressions were induced in dark and light conditions, 15 circadian rhythm genes were significantly associated with flowering time genes in the current transcriptome data set; LHY=late elongated hypocotyl, PIF3= phytochrome-interacting factor 3 , $P R R 7=$ pseudo-response regulator $7, P R R 5=$ pseudo-response regulator $5, P H Y B=$ phytochrome, $C R Y=$ cryptochrome, TOC1 $=$ timing of cab expression 1, HY5 = long hypocotyl 5, FT = flowering locus $T, G I=$ gigantea, $C O=C O N S T A N S, C H S=$ Chalcone and stilbene synthase, SPA1 = suppressor of phyA-105, ELF3 = early flowering 3.

\begin{tabular}{|c|c|c|c|c|}
\hline Code & Identity & Gene name & Biological function & $P$ value \\
\hline 1 & Phvul.006G086700 & $L H Y$ & Homeodomain-like superfamily protein & $5.24 \mathrm{E}-11$ \\
\hline 3 & Phvul.008G147400 & PRR7 & CCT motif family protein & $1.02 \mathrm{E}-03$ \\
\hline 5 & Phvul.010G153200 & $C R Y$ & DNA photolyase family protein & $4.65 \mathrm{E}-02$ \\
\hline 6 & Phvul.002G039100 & $\mathrm{CHS}$ & Chalcone and stilbene synthase family protein & $3.45 \mathrm{E}-03$ \\
\hline 7 & Phvul.003G188400 & TOC1 & CCT motif-containing response regulator protein & 4.60E-09 \\
\hline 10 & Phvul.008G257300 & PRR5 & CCT motif family protein & $1.96 \mathrm{E}-02$ \\
\hline 11 & Phvul.010G111200 & $S P A 1$ & SPA (suppressor of phyA-105) protein family & $1.77 \mathrm{E}-05$ \\
\hline 12 & Phvul.004G088300 & $G I$ & Gigantea protein $(\mathrm{GI})$ & $7.62 \mathrm{E}-04$ \\
\hline 13 & Phvul.001G097200 & $F T$ & PEBP (phosphatidylethanolamine-binding protein) family protein & $6.12 \mathrm{E}-04$ \\
\hline 14 & Phvul.006G005200 & $\mathrm{CO}$ & CONSTANS-like & $2.18 \mathrm{E}-04$ \\
\hline
\end{tabular}

Table 3. Expression patterns of eight differentially expressed genes related to flowering time in common bean. To evaluate functions and expression patterns of circadian clock-related genes, eight differentially expressed genes were found to be relate to flowering time, based on analyses using the Kyoto Encyclopedia of Genes and Genomes (KEGG) database; ELF3 = early flowering 3, PRR7 = pseudo-response regulator $7, P R R 5=$ pseudo-response regulator 5 , $L H Y=$ late elongated hypocotyl, TOC1 = timing of cab expression $1, G I=$ gigantea,$C O=$ constans, $F T=$ flowering locus $T$.

\begin{tabular}{llclc}
\hline & & & \multicolumn{2}{c}{ Regulation } \\
\cline { 4 - 5 } Code & Gene & Gene name & Light & Dark \\
\hline 1 & Phvul.010G142900 & ELF3 & Down & Down \\
2 & Phvul.008G257300 & PRR5 & Down & Down \\
3 & Phvul.008G147400 & PRR7 & Down & Down \\
4 & Phvul.006G086700 & LHY & Down & Up \\
5 & Phvul.003G188400 & TOC1 & Down & Up \\
6 & Phvul.004G088300, & $G I$ & Down & Down \\
& Phvul.007G083500 & & & \\
7 & Phvul.006G005200 & CO & Down & Up \\
8 & Phvul.001G097200 & $F T$ & Down & Down \\
\hline
\end{tabular}

libraries. Its expression in dark conditions was 2 -fold than in light conditions, whereas it was negatively regulated by the expression of the GI (Supplemental Fig. 2). The results suggest that $T O C 1$ plays a more important role than $L H Y$ in regulating the circadian clock rhythm in common bean. Notably, other genes involved in the molecular clock also were detected as being differentially regulated. TOC1 played the key role of transmitting signals to regulate the flowering process. TOC1 transmitted signals to downstream genes, and dark and light conditions could induce the upregulation and downregulation of TOC1 expression, respectively, thereby activating the flowering process in common bean (Supplemental Fig. 2). The circadian-regulated gene, $C O$, is a critical regulator of the pathway, and coordinates light and clock inputs in leaves to trigger the expression of florigen gene $F T$ (Suárez-López et al., 2001). In A. thaliana, an LD plant, the transcript peak of $C O$ mRNA occurs late in the day, but occurs after dusk in an SD plant (Suárez-López et al., 2001). In the present study, common bean is an SD plant and the transcript peak of the $C O$ mRNA is the exact opposite of the one in A. thaliana. We can infer that the expression pattern of $C O$ was influenced by photoperiod.

The number of reports for economically relevant traits in the common bean is still very limited. Kamfwa et al. (2015), using 237 genotypes of common beans genotyped for 5398 singlenucleotide polymorphisms (SNPs), found two significant SNPs for phenological traits days to flowering and days to maturity. These authors suggested the positional gene Phvul.001G221100, located on Pv01, as a candidate gene for controlling photoperiod sensitivity and flowering in common bean. In this study, Phvul.001G221100 was particularly upregulated under light conditions, and downregulated in dark conditions. But Phvul.001G221100 was not detected in the results of the GO term enrichment analysis. It was not observed to be significantly associated with flowering time genes in the current transcriptome data set. Moyses et al. (2018) investigated potential candidate genes in the region around seven significant SNPs. Three genes involved in the flowering pathways were identified, including Phvul.001G214500, Phvul.007G229300, and Phvul.010G142900.1 on Pv01, Pv07, and Pv10, respectively. In this study, Phvul.010G142900.1 observed ELF3 that exhibited downregulation in both light and dark conditions. It is proposed that, in the absence of ELF3 to gate light input, the clock is continually reset until the plants are returned to darkness. Thus, ELF3 might be predicted to be very important in maintaining the rhythm as days are lengthening toward summer and, indeed, elf3 mutants show an inability to adapt properly to LDs, becoming responsive rather than predictive in $A$. thaliana (Covington et al., 2001; Liu et al., 2001; McWatters et al., 2000). In the network, the circadian-regulated gene, $E L F 3$, is a key regulator of the pathway that coordinates light and circadian clock inputs in leaves to trigger the expression of downstream genes.

In summary, our analysis revealed that signaling molecules under LD or SD conditions induce changes in the expression of plant genes that participate in circadian rhythms, and facilitate the observation of a delayed flowering phenotype. In addition, our 
results illustrate how flowering time can be modulated by alternative transcript processing under different photoperiods. Our results highlight, to the best of our knowledge, novel biological and molecular aspects that could facilitate the understanding and identification of downstream responses in photoperiod regulation, and provide critical information on flowering time genes, which would facilitate further investigations on mechanisms of flower development. However, further studies of these flowering time-related genes are needed to better understand the network for regulating flowering time in common bean.

\section{Literature Cited}

Aidyn, M., C. Frédéric, and G. Coupland. 2002. Control of flowering time: Interacting pathways as a basis for diversity. Plant Cell 14:111-130.

Bhakta, M.S., S.A. Gezan, J.A. Clavijo Michelangeli, M. Carvalho, L. Zhang, and J.W. Jones. 2017. A predictive model for time-toflowering in the common bean based on QTL and environmental variables. Genes Genomes Genet. 7:3901-3912.

Cober, E.R. and J.W. Tanner. 1995. Performance of related indeterminate and tall determinate soybean lines in short-season areas. Crop Sci. 35:361-364.

Covington, M.F., S. Panda, X.L. Liu, C.A. Strayer, D.R. Wagner, and S.A. Kay. 2001. ELF3 modulates resetting of the circadian clock in Arabidopsis. Plant Cell 13:1305-1315.

Doust, A.N., M. Mauro-Herrera, J.G. Hodge, and J. Stromski. 2017. The C4 model grass Setaria is a short day plant with secondary long day genetic regulation. Front. Plant Sci. 8:1062.

Fujiwara, S., A. Oda, and R. Yoshida. 2008. Circadian clock proteins LHY and CCA1 regulate SVP protein accumulation to control flowering in Arabidopsis. Plant Cell 20:2960-2971.

Gepts, P. and D.G. Debouck. 1991. Origin, domestication, and evolution of the common bean (Phaseolus vulgaris L.), p. 7-53. In: A. van Schoonhoven and O. Voysest (eds.). Common beans: Research for crop improvement. Commonwealth Agricultural Bureaux Intl., Wallingford, UK.

Gu, W., J. Zhu, D. Wallace, S. Singh, and N. Weeden. 1998. Analysis of genes controlling photoperiod sensitivity in common bean using DNA markers. Euphytica 102:125-132.

Itoh, H., Y. Nonoue, M. Yano, and T. Izawa. 2010. A pair of floral regulators sets critical day length for $\mathrm{Hd} 3$ a florigen expression in rice. Nat. Genet. 42:635-638.

Kojima, S., Y. Takahashi, Y. Kobayashi, L. Monna, and T. Sasaki. 2002. Hd3a, a rice ortholog of the Arabidopsis FT gene, promotes transition to flowering downstream of Hd1 under short-day conditions. Plant Cell Physiol. 43:1096-1105.

Kornegay, J., J.W. White, J.R. Dominguez, G. Tejada, and C. Cajiao. 1993. Inheritance of photoperiod response in Andean and Mesoamerican common bean. Crop Sci. 33:977-984.

Kwak, M., D. Velasco, and P. Gepts. 2008. Mapping homologous sequences for determinacy and photoperiod sensitivity in common bean (Phaseolus vulgaris). J. Hered. 99:283-291.

Kamfwa, K., K.A. Cichy, and J.D. Kelly. 2015. Genome-wide association study of agronomic traits in common bean. Plant Genome $8: 1-12$.
Kitae, S., K.H. Chul, S. Seungho, K. Kyung-Hee, M. Jun-Cheol, and K.J. Yoo. 2017. Transcriptome analysis of flowering time genes under drought stress in maize leaves. Front. Plant Sci. 8:267.

Liu, X.L., M.F. Covington, C. Fankhauser, J. Chory, and D.R. Wagner. 2001. ELF3 encodes a circadian clock-regulated nuclear protein that functions in an Arabidopsis PHYB signal transduction pathway. Plant Cell 13:1293-1304.

Lee, S. and G. An. 2007. Diversified mechanisms for regulating flowering time in a short-day plant rice. J. Plant Biol. 50:241-248.

Li, R., C. Yu, Y. Li, T.W. Lam, S.M. Yiu, K. Kristiansen, and J. Wang. 2009. SOAP2: An improved ultrafast tool for short read alignment. Bioinformatics 25:1966-1967.

Lu, S.X., S.M. Knowles, C. Andronis, M.S. Ong, and E.M. Tobin. 2009. Circadian clock associated1 and late elongated hypocotyl function synergistically in the circadian clock of Arabidopsis. Plant Physiol. 150:834-843.

McWatters, H.G., R.M. Bastow, A. Hall, and A.J. Millar. 2000. The ELF3 zeitnehmer regulates light signalling to the circadian clock. Nature 408:716-720.

Moyses, N., A.C.C. Nascimento, and F.F.E. Silva. 2018. Quantile regression for genome-wide association study of flowering timerelated traits in common bean. PLoS One 13:0190303.

Nagel, D.H. and S.A. Kay. 2012. Complexity in the wiring and regulation of plant circadian networks. Curr. Biol. 22:648-657.

Putterill, J., R. Laurie, and R. Macknight. 2004. It's time to flower: The genetic control of flowering time. BioEssays 26:363-373.

Rajkumar, A.P., P. Qvist, R. Lazarus, F. Lescai, J. Ju, M. Nyegaard, O. Mors, A.D. Børglum, Q. Li, and J.H. Christensen. 2015. Experimental validation of methods for differential gene expression analysis and sample pooling in RNA-seq. BMC Genomics 16:548.

Salome, P.A., K. Bomblies, R.A.E. Laitinen, L. Yant, and R. Mott. 2011. Genetic architecture of flowering-time variation in Arabidopsis thaliana. Genetics 188:421-433.

Simpson, G.G. and C. Dean. 2002. Arabidopsis, the Rosetta Stone of flowering time. Science 296:285-289.

Suárez-López, P., K. Wheatley, F. Robson, H. Onouchi, F. Valverde, and G. Coupland. 2001. CONSTANS mediates between the circadian clock and the control of flowering in Arabidopsis. Nature 410:1116-1120.

Tamaki, S., S. Matsuo, H. Wong, S. Yokoi, and K. Shimamoto. 2007. $\mathrm{Hd} 3$ a protein is a mobile flowering signal in rice. Science 316:10331037.

Wallace, D.H., P.A. Gniffke, P.N. Masaya, and R.W. Zobel. 1991. Photoperiod, temperature, and interaction effects on days and nodes required for flowering of bean. J. Amer. Soc. Hort. Sci. 116:534-543. White, J. and D. Laing. 1989. Photoperiod response of flowering in diverse genotypes of common bean (Phaseolus vulgaris). Field Crops Res. 22:113-128.

White, J., J. Kornegay, and C. Cajiao. 1996. Inheritance of temperature sensitivity of the photoperiod response in common bean (Phaseolus vulgaris L.). Euphytica 91:5-8.

Yanovsky, M. and S. Kay. 2002. Molecular basis of seasonal time measurement in Arabidopsis. Nature 419:308-312.

Zhuo, J.W., P. Bao-Ping, and F. Yong-Fu. 2010. Bioinformatics analysis of the TOC1 homologs in soybean genome. J. Henan Agr. Sci. 56:14-19. 


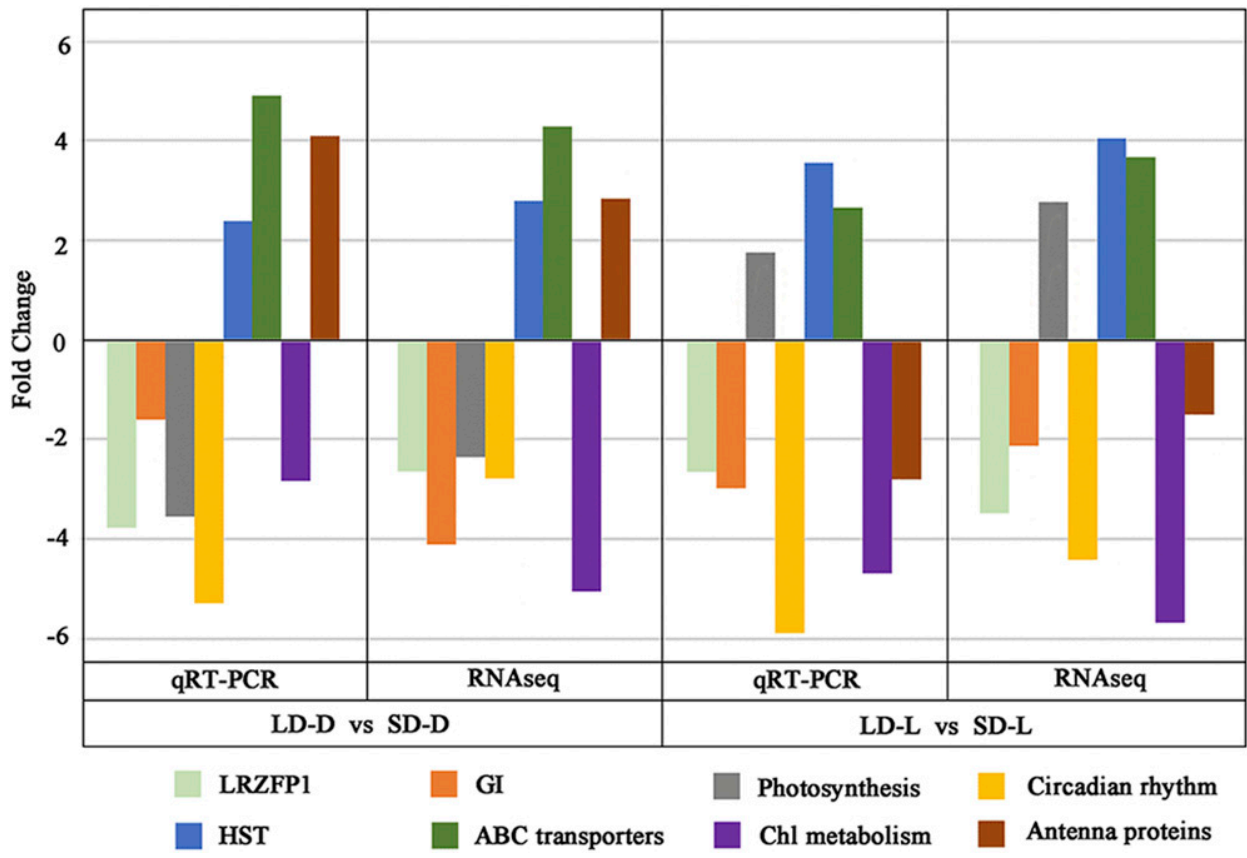

Supplemental Fig. 1. Quantitative reverse transcription polymerase chain reaction (qRT-PCR) validation of the relative expression levels of genes selected from the RNA-sequencing (RNA-seq) analysis of common bean-photoperiod \{long-day [LD (16/8 h light/dark)] and short-day [SD (8/16 h light/dark)] interaction. Expression profiles of eight genes (color coded) as determined by qRT-PCR and RNA-seq. The $x$-axis indicates two groups of comparisons: LD-D vs. SD-D. and LD-L vs. SD-L [LD-L (0600 HR), LD-D (1400 HR), SD-L (0600 HR), and SD-D (1400 HR)]. The $y$-axis shows the fold change increases/decreases in expression levels of genes; $L R Z F P 1=L R$ zinc finger protein, $G I=$ gigantea, $H S T=$ homogentisate solanesyl transferase . 


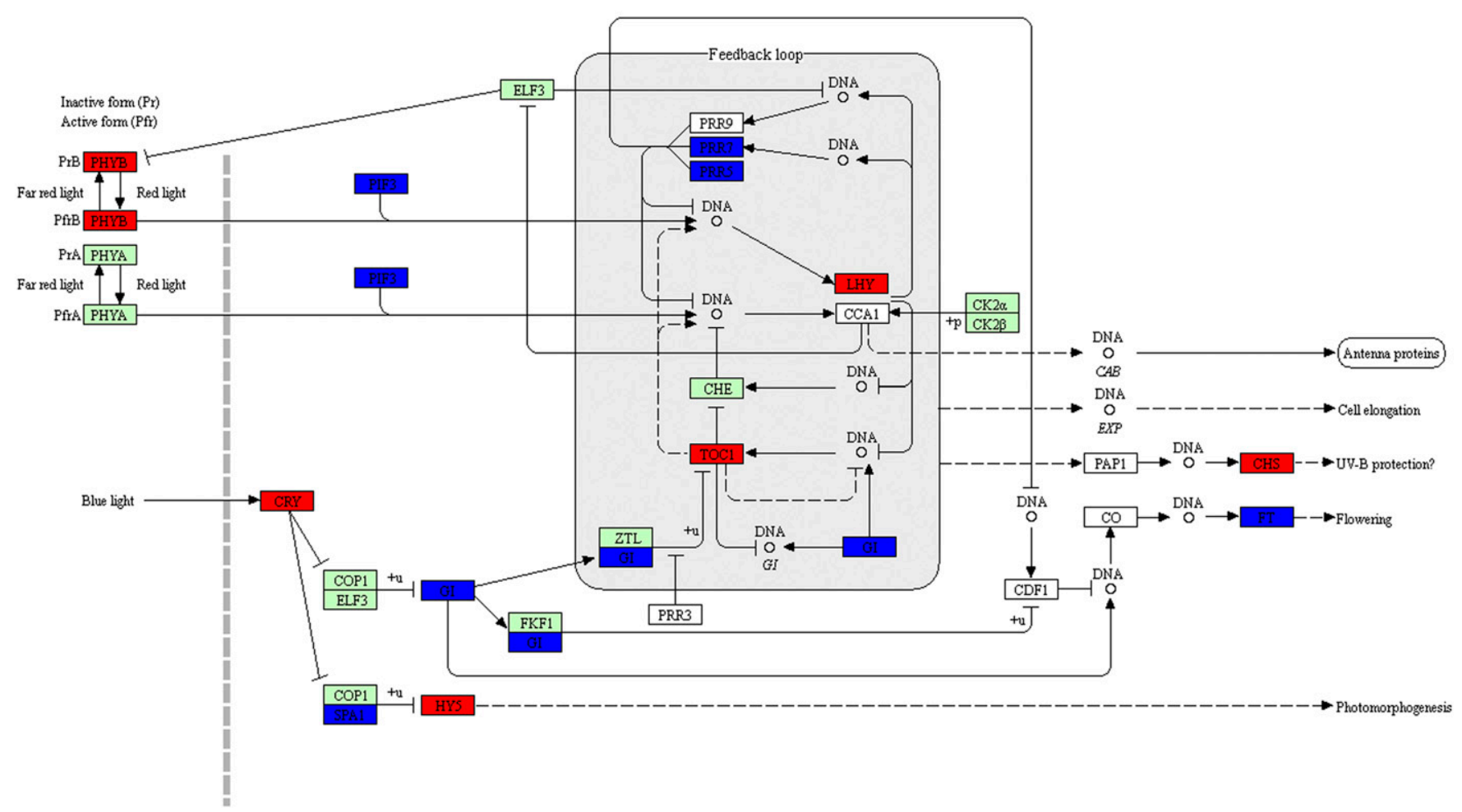

Supplemental Fig. 2. Kyoto Encyclopedia of Genes and Genomes (KEGG) pathway. Regulation of circadian rhythm genes in response to photoperiod in common bean. Schematic representation of key circadian clock components identified in common bean and their transcriptional interactions (red: upregulation, blue: downregulation). $L H Y=$ late elongated hypocotyl, $P I F 3=$ phytochrome-interacting factor $3, P R R 9=$ pseudo-response regulator $9, P R R 7=p s e u d o$-response regulator $7, P R R 5=$ pseudo-response regulator $5, P H Y B=$ phytochrome, $C R Y=$ cryptochrome, $T O C 1=$ timing of cab expression $1, H Y 5=$ long hypocotyl $5, F T=$ flowering locus $T, G I=$ gigantea, ELF3 = early flowering 3, CO = constans, $P H Y A=$ phytochrome $A, C O P 1=E 3$ ubiquitin-protein ligase RFWD2, FKF1 = flavinbinding kelch repeat F-box protein 1, ZTL = clock-associated PAS protein, CHE = transcription factor TCP21, CCA1 = circadian clock associated 1, CK2 = casein kinase II, CHS = chalcone synthase, PAPI = transcription factor MYB75, CDF1 = Dof zinc finger protein DOF5.5. 
Supplemental Table 1. List of common bean cultivars, growth habits, and phenotypes used in this study. A total of 215 common bean cultivars

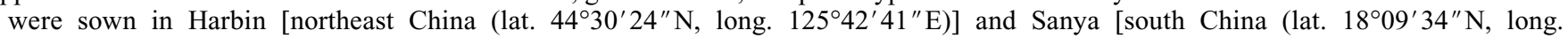
$\left.\left.108^{\circ} 56^{\prime} 30^{\prime \prime} \mathrm{E}\right)\right]$ on 21 May and 1 Nov. 2017, respectively.

\begin{tabular}{|c|c|c|c|c|}
\hline \multirow[b]{2}{*}{ Code } & \multirow[b]{2}{*}{ Cultivar } & \multirow[b]{2}{*}{ Growth habit } & \multicolumn{2}{|c|}{ Time to flowering (d) } \\
\hline & & & Harbin & Sanya \\
\hline 2 & Xiang jiao you dou & Indeterminate viny & 51 & 38 \\
\hline 4 & Qi ji & Indeterminate viny & 41 & 42 \\
\hline 5 & Zao feng & Indeterminate viny & 39 & 38 \\
\hline 6 & Kuai jian & Indeterminate viny & 40 & 37 \\
\hline 9 & Jiu yue & Determinate brush & 41 & 39 \\
\hline 10 & Jia you dou & Determinate brush & 39 & 37 \\
\hline 11 & Yi ke song & Determinate brush & 40 & 38 \\
\hline 12 & Dan se qiu & Determinate brush & 40 & 39 \\
\hline 13 & $73-8$ & Determinate brush & 40 & 34 \\
\hline 18 & Wu jin 6 & Indeterminate viny & 44 & 42 \\
\hline 19 & F0119 & Indeterminate viny & 47 & 45 \\
\hline 20 & F0678 & Determinate brush & 42 & 39 \\
\hline 21 & F0705 & Determinate brush & 42 & 40 \\
\hline 22 & F1736 & Determinate brush & 47 & 45 \\
\hline 23 & F2320 & Determinate brush & 42 & 38 \\
\hline 24 & F3420 & Indeterminate viny & 54 & 37 \\
\hline 25 & F4150 & Indeterminate viny & 49 & 50 \\
\hline 26 & F4226 & Indeterminate viny & 50 & 48 \\
\hline 27 & BE221-1 & Determinate brush & 41 & 38 \\
\hline 28 & Shuang feng NO.3 & Indeterminate viny & 41 & 39 \\
\hline 36 & Shi li chang & Indeterminate viny & 49 & 45 \\
\hline 37 & Da ma zhang & Indeterminate viny & 49 & 47 \\
\hline 38 & $851-923-9$ & Indeterminate viny & 42 & 39 \\
\hline 39 & $97-5$ & Indeterminate viny & 42 & 40 \\
\hline 40 & Yin bai tiao & Indeterminate viny & 42 & 41 \\
\hline 41 & Zao man jia & Indeterminate viny & 43 & 38 \\
\hline 42 & Qing feng di dou & Determinate brush & 40 & 40 \\
\hline 43 & Qing dao dou & Determinate brush & 40 & 40 \\
\hline 44 & $81-6$ & Determinate brush & 41 & 39 \\
\hline 45 & Shuang sheng jia dou & Determinate brush & 40 & 40 \\
\hline 46 & Tian ma di dou & Determinate brush & 40 & 38 \\
\hline 47 & Shuang sheng di dou & Determinate brush & 41 & 39 \\
\hline 48 & $5991(81-6)$ & Determinate brush & 41 & 37 \\
\hline 49 & No. 16 & Indeterminate viny & 51 & 49 \\
\hline 50 & $4-10$ & Indeterminate viny & 49 & 49 \\
\hline 51 & Tu zi fan bai yan & Indeterminate viny & 50 & 38 \\
\hline 52 & $96-9-17$ & Indeterminate viny & 41 & 41 \\
\hline 53 & $96-8-50$ & Indeterminate viny & 44 & 40 \\
\hline 54 & $96-8-14$ & Indeterminate viny & 49 & 47 \\
\hline 55 & $96-8-7$ & Indeterminate viny & 41 & 40 \\
\hline
\end{tabular}




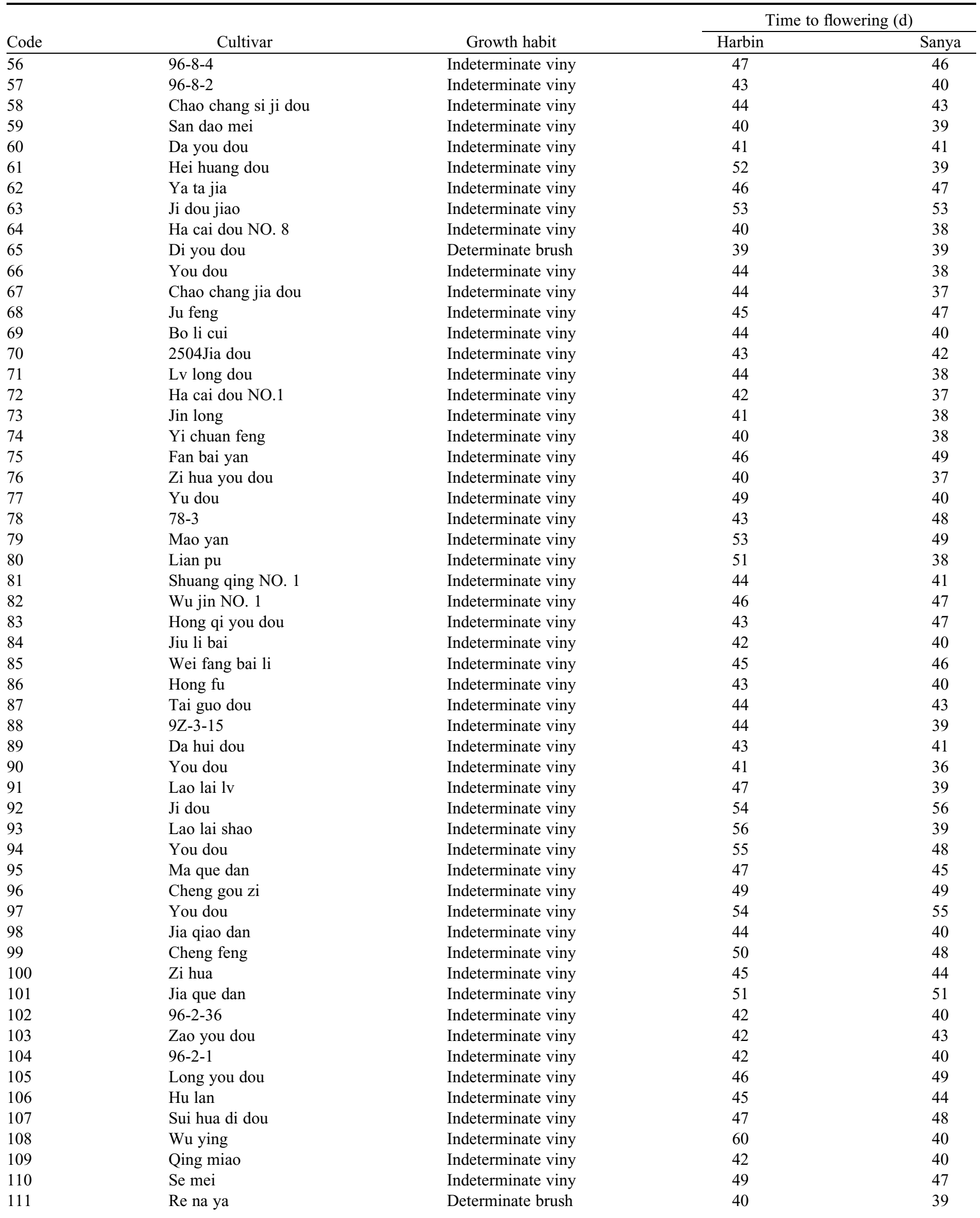


Supplemental Table 1. Continued.

\begin{tabular}{|c|c|c|c|c|}
\hline \multirow[b]{2}{*}{ Code } & \multirow[b]{2}{*}{ Cultivar } & \multirow[b]{2}{*}{ Growth habit } & \multicolumn{2}{|c|}{ Time to flowering (d) } \\
\hline & & & Harbin & Sanya \\
\hline 112 & Ai zi dou & Determinate brush & 41 & 40 \\
\hline 113 & Ai huang dou & Determinate brush & 38 & 39 \\
\hline 115 & Gong ji zhe & Determinate brush & 40 & 40 \\
\hline 116 & P-15-61 Borlotlo U & Determinate brush & 39 & 39 \\
\hline 119 & Hua pi dou & Indeterminate viny & 61 & 59 \\
\hline 120 & Yu long & Indeterminate viny & 50 & 51 \\
\hline 121 & Chi yu & Indeterminate viny & 44 & 43 \\
\hline 122 & Jia huang & Indeterminate viny & 43 & 40 \\
\hline 123 & Hong guang & Indeterminate viny & 43 & 39 \\
\hline 124 & Feng lan & Indeterminate viny & 43 & 40 \\
\hline 128 & $\mathrm{Xu}$ shen & Determinate brush & 41 & 40 \\
\hline 129 & Qi jiang & Determinate brush & 41 & 39 \\
\hline 130 & Guang hui & Determinate brush & 41 & 34 \\
\hline 131 & Yin bao & Determinate brush & 42 & 36 \\
\hline 132 & A95-24 & Determinate brush & 41 & 38 \\
\hline 133 & A18-13 & Indeterminate viny & 41 & 41 \\
\hline 134 & B96-1 & Determinate brush & 40 & 37 \\
\hline 135 & Qing lang & Determinate brush & 41 & 42 \\
\hline 136 & Wu shi & Determinate brush & 42 & 39 \\
\hline 137 & Li ze & Determinate brush & 43 & 40 \\
\hline 138 & Sheng qi & Determinate brush & 42 & 41 \\
\hline 139 & Fang zheng & Determinate brush & 40 & 39 \\
\hline 147 & CIAT-107 & Determinate brush & 41 & 41 \\
\hline 148 & DDF-309 & Indeterminate viny & 46 & 50 \\
\hline 149 & E-MAR & Indeterminate viny & 46 & 43 \\
\hline 150 & GER & Indeterminate viny & 46 & 45 \\
\hline 151 & JAX & Indeterminate viny & 45 & 48 \\
\hline 152 & VAX & Indeterminate viny & 47 & 47 \\
\hline 153 & VAXL & Indeterminate viny & 46 & 48 \\
\hline 154 & VAT & Indeterminate viny & 46 & 45 \\
\hline 155 & XAV309 & Indeterminate viny & 46 & 44 \\
\hline 156 & Z-MAR & Indeterminate viny & 51 & 50 \\
\hline 157 & G0446 & Determinate brush & 40 & 43 \\
\hline 158 & G173441 & Indeterminate viny & 51 & 50 \\
\hline 159 & Qi san ba & Indeterminate viny & 40 & 40 \\
\hline 160 & Jiu li hong & Indeterminate viny & 40 & 38 \\
\hline 161 & Yi li wang & Indeterminate viny & 40 & 41 \\
\hline 162 & Hei cha ma zhang & Indeterminate viny & 41 & 40 \\
\hline 163 & Xin zi cha & Indeterminate viny & 40 & 42 \\
\hline 164 & $\mathrm{Zi}$ cha you dou & Indeterminate viny & 41 & 42 \\
\hline 165 & Chao zao jia dou & Indeterminate viny & 42 & 41 \\
\hline 166 & Jia NO.57 & Indeterminate viny & 42 & 42 \\
\hline 167 & Xiao cha ba & Indeterminate viny & 43 & 43 \\
\hline
\end{tabular}


Supplemental Table 1. Continued.

\begin{tabular}{|c|c|c|c|c|}
\hline \multirow[b]{2}{*}{ Code } & \multirow[b]{2}{*}{ Cultivar } & \multirow[b]{2}{*}{ Growth habit } & \multicolumn{2}{|c|}{ Time to flowering $(\mathrm{d})$} \\
\hline & & & Harbin & Sanya \\
\hline 168 & Fan bai yan & Determinate brush & 41 & 40 \\
\hline 169 & Hong yu & Determinate brush & 42 & 42 \\
\hline 171 & Qing lv & Indeterminate viny & 42 & 43 \\
\hline 172 & D312G-4-3 & Indeterminate viny & 41 & 42 \\
\hline 175 & Yu shu you dou & Indeterminate viny & 43 & 43 \\
\hline 176 & Da que dan & Indeterminate viny & 43 & 43 \\
\hline 177 & Lv jiao hei zhen zhu & Indeterminate viny & 43 & 45 \\
\hline 178 & D312G-9-4-2 & Determinate brush & 42 & 41 \\
\hline 179 & D312G-3 & Indeterminate viny & 41 & 42 \\
\hline 180 & D312G-111 & Indeterminate viny & 43 & 43 \\
\hline 184 & $21 R G-1-5-1-2$ & Indeterminate viny & 42 & 42 \\
\hline 185 & $35 \mathrm{M}-9-1$ & Indeterminate viny & 39 & 43 \\
\hline 186 & GH15-3-2-1 & Indeterminate viny & 40 & 40 \\
\hline 187 & HG-5-1 & Indeterminate viny & 40 & 41 \\
\hline 188 & $16-9-1-3$ & Indeterminate viny & 41 & 38 \\
\hline 189 & $61-11-2$ & Indeterminate viny & 41 & 39 \\
\hline 190 & GZG & Indeterminate viny & 39 & 40 \\
\hline 191 & Jiao long338 & Indeterminate viny & 41 & 44 \\
\hline 192 & Hong bian bian & Indeterminate viny & 42 & 42 \\
\hline 193 & Hong ni & Indeterminate viny & 39 & 39 \\
\hline 194 & Lu wang No.2 & Indeterminate viny & 41 & 38 \\
\hline 195 & Huang se wu jia & Indeterminate viny & 41 & 42 \\
\hline 203 & Xin peng shi wang & Indeterminate viny & 41 & 43 \\
\hline 204 & Ji feng & Indeterminate viny & 41 & 48 \\
\hline 205 & Huang jiao zhen zhu & Indeterminate viny & 41 & 40 \\
\hline 206 & Qing long wu jin & Indeterminate viny & 42 & 43 \\
\hline 207 & Xi nuo jia dou & Indeterminate viny & 41 & 41 \\
\hline 208 & Bai li qing jia & Indeterminate viny & 40 & 43 \\
\hline 209 & D312G-4-16 & Determinate brush & 41 & 42 \\
\hline 210 & Yi ke song & Indeterminate viny & 41 & 41 \\
\hline 211 & Jiu yue qing & Indeterminate viny & 46 & 42 \\
\hline 212 & Long yan & Indeterminate viny & 44 & 41 \\
\hline 213 & HG911 & Indeterminate viny & 46 & 45 \\
\hline 214 & Ha you san & Indeterminate viny & 40 & 39 \\
\hline 215 & Wan xia & Indeterminate viny & 42 & 41 \\
\hline
\end{tabular}


Supplemental Table 2. List of primers used to amplify the selected genes for quantitative reverse transcription polymerase chain reaction (qRTPCR) analyses. Eight genes with varying expression patterns revealed through RNA-sequencing were randomly selected for validation by qRT-PCR. RNA extracted from the leaves of the three independent biological replicates of the different sampling times were used for qRTPCR validation. Gene copy specific primers for qRT-PCR were designed based on the corresponding sequences using Primer6; $L R Z F P 1=L R$ zinc finger protein, GI = gigantea, HST = homogentisate solanesyl transferase.

\begin{tabular}{lllll}
\hline Code & \multicolumn{1}{c}{ Gene name } & \multicolumn{1}{c}{ Identity } & \multicolumn{1}{c}{ Forward primer $\left(5^{\prime}-3^{\prime}\right)$} & Reverse primer $\left(5^{\prime}-3^{\prime}\right)$ \\
\hline 1 & LRZFPl & Phvul.011G168500 & TCGTCCTCTGTCAAGTCA & GCAGTATAACCACCACCAT \\
2 & GI & Phvul.007G083500 & GCCAAGAGGTGAAGATAACT & CAGCAGCCAGAACATAAGA \\
3 & Photoperiod & Phvul.009G225500 & AACTGCTAAGTGCGTGTT & GTAGTGTTACCTAATCCAAGAG \\
4 & Circadian rhythm & Phvul.009G045000 & CAACCTCACACTCTCAACA & GAACTTGGACTTGGCATTG \\
5 & HST & Phvul.001G265300 & CTGAGAGTGCTGGTGTTC & ATTGCGGTAGTATGCTGTAA \\
6 & ABC transporters & Phvul.004G027800 & TGGCACATCGTCTATCAAC & GTCTTGGCAATGAGTTCTTC \\
7 & Chl metabolism & Phvul.008G185100 & TGAATTAAGAACCGCAGGAT & AGCCGTGTGGATTATTGAG \\
8 & Antenna proteins & Phvul.004G071500 & CTGCCAACACATTGATGAG & GCATCCAGTCAGAAGTCAT \\
\hline
\end{tabular}

